\title{
A Radiation Hybrid Map of the Proximal Short Arm of the Human X Chromosome Spanning Incontinentia Pigmenti 1 (IP1) Translocation Breakpoints
}

\author{
Jerome L. Gorski, ${ }^{*} \dagger{ }^{1}$ Michael Boehnke, $\ddagger$ Eric L. Reyner, ${ }^{*}$ and Eric N. Burright† \\ Departments of *Pediatrics and Communicable Diseases, $¥$ Biostatistics, and †Human Genetics, \\ University of Michigan, Ann Arbor, Michigan 48109-0688
}

Received May 6, 1992; revised July 27, 1992

\begin{abstract}
Radiation hybrid mapping was used in combination with physical mapping techniques to order and estimate distances between 14 loci in the proximal region of the short arm of the human $X$ chromosome. A panel of radiation hybrids containing human $\mathrm{X}$-chromosomal fragments was generated from a Chinese hamster-human cell hybrid containing an $X$ chromosome as its only human DNA. Sixty-seven radiation hybrids were screened by Southern hybridization with sets of probes that mapped to the region $X p 11.4-X c e n$ to generate a radiation hybrid map of the area. A physical map of 14 loci was constructed based on the segregation of the loci in the hybrid clones. Using pulsed-field gel electrophoresis (PFGE) analyses and a somatic cell hybrid mapping panel containing naturally occurring $X$; autosome translocations, the order of the 14 loci was verified and the loci nearest to the $\mathrm{X}$-chromosomal translocation breakpoints associated with the disease incontinentia pigmenti 1 (IP1) were identified. The radiation hybrid panel will be useful as a mapping resource for determining the location, order, and distances between other genes and polymorphic loci in this region as well as for generating additional region-specific DNA markers. (C) 1992 Academic Press, Inc.
\end{abstract}

\section{INTRODUCTION}

A physical map of a targeted chromosomal region is an essential prerequisite for the positional cloning of human disease-associated genes. The construction and characterization of somatic cell hybrids containing defined human chromosomal fragments has been instrumental in the development of high-resolution physical maps. As part of an effort to clone the incontinentia pigmenti 1 (IP1) locus and improve the resolution of a regional physical map, we sought a strategy to order and

\footnotetext{
${ }^{1}$ To whom correspondence and reprint requests should be addressed at Division of Pediatric Genetics, Department of Pediatrics, Rm 3570 Medical Science Research Building II, P.O. Box 0688, University of Michigan Medical Center, Ann Arbor, MI 48109-0688.
}

determine the distances between loci previously mapped in the vicinity of the IP1 X-chromosomal translocation breakpoints.

The IP1 locus has been mapped to region Xp11.21 by cytologic (Gilgenkrantz et al., 1985; Hodgson et al., 1985; Kajii et al., 1985; Cannizzaro and Hecht, 1987; Crolla et al., 1989) and molecular (Gorski et al., 1991) analyses. Our previous mapping strategies relied on naturally occurring disease-specific translocations; these resources provided a method of mapping DNA markers relative to IP1 translocation breakpoints and assisted in constructing a primary physical map of the IP1 locus. This map indicated that the IP1 X-chromosomal translocation breakpoints were separated by at least $1250 \mathrm{~kb}$ (Gorski et al., 1991). Subsequent studies showed that this region was at least twice that size (Gorski et al., 1992); these results suggested that it would be difficult to determine precisely an order for and distance between the mapped loci solely by PFGE analysis. Therefore, we sought an alternative method for constructing a physical map of the IP1 region.

Radiation hybrid mapping is a powerful somatic cell genetic technique for ordering DNA markers which extend over millions of basepairs of DNA (Cox et al., 1990). In this method, a lethal dose of radiation is used to cleave a human chromosome in a somatic cell hybrid into numerous fragments. Human chromosome fragments are subsequently retained nonselectively in a second rodent cell following cell fusion; the resulting hybrid clones are then screened for the presence or absence of human chromosome-specific loci. The closer two markers are on a chromosome, the less likely they will be separated by radiation fragmentation. By analyzing the cosegregation of the various loci in hybrid clones, a physical map can be constructed (Cox et al., 1990; Warrington et al., 1991; Ceccherini et al., 1992).

To better define the region flanking the IP1 X-chromosomal translocation breakpoints, we used radiation hybrid mapping and molecular mapping techniques to construct a physical map of 14 loci in the proximal region of $\mathrm{Xp}$, including loci mapped to a region between 
IP1 breakpoints, loci immediately distal to the IP1 breakpoints within region $\mathrm{Xp} 11.21$, and a candidate gene for X-linked sideroblastic anemia (ALAS2) mapped to distal Xp11.21 (Cotter et al., 1992; Gorski et al., 1992).

A panel of radiation hybrids containing fragments of a human $\mathrm{X}$ chromosome was generated from an X-only somatic cell hybrid we previously had used to construct a physical map of the region (Gorski et al., 1991, 1992); this provided a means of calibrating the radiation hybrid map and relating physical distances determined by PFGE to kilobasepairs of DNA. By analyzing the segregation of the 14 markers in the hybrid clones, we were able to construct a physical map defining the position, order, and distances between loci. These results, in conjunction with the results of physical mapping analyses, provided a means to construct a consensus map of the region. The resultant map predicted close physical linkage between several loci and identified those loci in closest proximity to the IP1 X-chromosomal translocation breakpoints. This map provides a starting point for establishing contigs of YAC and cosmid clones spanning the IP1 breakpoints and a means of ordering additional genes and loci mapped to this region.

\section{MATERIALS AND METHODS}

Radiation hybrids and somatic cell hybrids. Radiation hybrids (RHs) containing portions of a human $\mathrm{X}$ chromosome were constructed by irradiating cell line GM06318, a human-hamster hybrid containing a single, structurally intact, human $\mathrm{X}$ chromosome (Coriell Medical Institute, Camden, NJ) and performing a polyethylene glycol-mediated fusion with thymidine kinase deficient (TK-) hamster cell line a23 (Westerveld et al., 1971). RHs were constructed as described by Cox et al. (1989) with minor modifications; $2 \times 10^{7}$ washed GM06318 cells were resuspended in $10 \mathrm{ml}$ of serum-free DMEM, placed in a $25-\mathrm{cm}^{2}$ flask on ice, and exposed to 8000 rads of irradiation using a ${ }^{137} \mathrm{Ce}$ source at a rate of $265 \mathrm{rads} / \mathrm{min}$. These cells were fused to $2 \times 10^{7}$ a23 cells as described previously (Gorski et al., 1989). Cells were plated at a density of $10^{6}$ recipient cells $/ 100-\mathrm{mm}$ tissue culture dish. RHs expressing the Chinese hamster TK gene from cell line GM06318 were selected in medium containing hypoxanthine, aminopterin, and thymidine (HAT). A total of 67 radiation hybrid clones were isolated; all were used for the described analyses.

An IP1 somatic cell hybrid mapping panel has been described previously (Gorski et al., 1989, 1991). Briefly, C9-5, B13-3, and C17-1D3 are human hamster hybrid cell lines and CLX17-A12 is a human-mouse hybrid cell line constructed from Epstein-Barr virus-transformed lymphoblastoid cell lines derived from three unrelated female patients with nonfamilial IP. Hybrids C9-5 and B13-3 contain the translocated $\mathrm{X}$ chromosome $\mathrm{t}(\mathrm{X} ; 9)(\mathrm{p} 11.21 ; \mathrm{q} 34.3)$ (Gilgenkrantz et al., 1985) and $\mathrm{t}(\mathrm{X} ; 13)(\mathrm{p} 11.21 ; \mathrm{q} 12.3)$ (Kajii et $a l$, 1985), respectively; hybrid C17$1 \mathrm{D} 3$ contains the derivative $\mathrm{X}$ chromosome, and hybrid CLX17-A12 contains the derivative 17 chromosome $t(X ; 17)(\mathrm{p} 11 ; \mathrm{p} 11)$ (Hodgson et al., 1985). Hybrid A63-1A contains the translocation chromosome $\mathrm{t}(\mathrm{X} ; 20)(20 \mathrm{pter}-\mathrm{q} 11.1: \mathrm{Xq11.1-Xqter})$; hybrid A48-1Fa contains the translocation chromosome $t(X ; 11)(11 p t e r-11 c e n:: X c e n-X q t e r)$ (Mahtani and Willard, 1988). Hybrid GM10501 contains the translocation chromosome $t(X ; 17)(17 q t e r-17 q 11.2:: X p 11.21-X q t e r)$ (Lafreniere et al., 1991) (Coriell Medical Institute).

Multipoint mapping of radiation hybrid data. $\mathrm{RH}$ mapping data were analyzed using the multipoint maximum likelihood method of Boehnke et al. (1991). This method assumes that X-ray breakage occurs randomly along a chromosome and that the resulting fragments are independently retained in a $\mathrm{RH}$. In the $N$-locus case, the likeli-
TABLE 1

DNA Fragments Used as Probes

\begin{tabular}{|c|c|c|c|}
\hline Probe & Locus & Fragment & $\begin{array}{l}\mathrm{X} \text {-chromosomal } \\
\text { regional assignment }\end{array}$ \\
\hline ALAS2 & & 1.8-kb EcoRI & $\mathrm{p} 11.21$ \\
\hline MTHFDL1 & & 2.0-kb EcoRI & $\mathrm{p} 11.21^{\circ}$ \\
\hline 2S/pEa84 & DXS706 & $1.0-\mathrm{kb} S a u 3 \mathrm{~A}$ & $\mathrm{p} 11.21^{a}$ \\
\hline $3 \mathrm{~S}$ & DX\$705 & 0.9-kb Sau3A & $\mathrm{p} 11.21^{a}$ \\
\hline p58-1 & DXS14 & 1.2-kb HindIII & $\mathrm{p} 11.21^{a}$ \\
\hline $30 \mathrm{C}_{\mathrm{E}} 2.8$ & DXS370 & 2.8-kb EcoRI & $\mathrm{p} 11.21^{a}$ \\
\hline $\operatorname{cpX210}$ & DXS422 & 0.3-kb EcoRI/HindIII & $\mathrm{p} 11.21^{\circ}$ \\
\hline $\mathrm{RX}-66$ & DXS343 & 1.2-kb Rsal & $\mathrm{p} 11.21^{a}$ \\
\hline $\mathrm{KZO}-48$ & DXS323 & 0.8-kb EcoRI/HhaI & p11.21 \\
\hline $2: 30^{b}$ & DXS741 & 1.4-kb Notl/HindIII & $\mathrm{p} 11.21^{b}$ \\
\hline $49 \mathrm{~A}^{\mathrm{c}}$ & DXS740 & $0.8-\mathrm{kb} R s a \mathrm{I}$ & $\mathrm{p} 11.21^{c}$ \\
\hline pHOTC & OTC & 1.4-kb EcoRI & $\mathrm{p} 11.4$ \\
\hline pTAK8a & DXS146 & 1.1-kb HindIII/BamHI & p11.21 \\
\hline pBamX7 & DXZ1 & 2.0-kb HindIII/BamHI & cen \\
\hline
\end{tabular}

Note. Locus identifications, regional assignments, and probe references are those of Human Gene Mapping 10.5 (Davies et al., 1990) and those previously reported (Lafreniere et al., 1991; Cotter et al., 1992; Gorski et al., 1992).

${ }^{a}$ Localized to a region between IP1 X-chromosomal translocation breakpoints (Gorski et al., 1991, 1992).

${ }^{b}$ NotI linking clone (Arenstorf et al., 1991); regional assignment determined by mapping data reported in the present work.

${ }^{c}$ IRS-PCR product amplified from a PFGE fractionated restriction fragment and mapped to region between IP1 breakpoints within Xp11.21 (Burright and Gorski, 1992).

hood of the RH data is a function of the $N-1$ breakage probabilities between adjacent loci and one or more fragment retention probabilities. This method makes use of data on all loci simultaneously, including information on partially typed hybrids.

To describe the different fragment retention probability models, let $r_{i j}$ be the probability of retaining a fragment on which only loci $i<i+1$ $<\cdots<j$ are present. We considered two such models. In the first, all fragment retention probabilities were assumed to be equal $\left(r_{i j}=r\right.$ for all $i \leqslant j$ ). In the second, we allowed for a centromeric effect by setting $r_{1 j}=r_{1}$ and $r_{i j}=r_{2}(1<i \leqslant j)$. Use of this second model was suggested by the decrease in observed locus retention probabilities with increasing distance from the centromere (see Results).

For each model, and for a given locus order, breakage and retention probabilities were estimated by those values that maximized the likelihood for the RH mapping data. Orders were compared by their maximum likelihoods, the order with the largest maximum likelihood being best supported by the data. For a given order, fragment retention models were compared by likelihood ratio tests.

Since it was not practical to consider explicitly all 14!/2 (equal retention) or 14 ! (centromeric retention) orders for the 14 loci, we used a stepwise locus ordering algorithm to identify the best locus order (Boehnke et al., 1991). This algorithm builds locus orders by adding one locus at a time. At each stage, it keeps under consideration those partial locus orders no more than $K$ times less likely than the current best partial locus order. We carried out stepwise locus ordering for both the equal and centromeric models for the fourteen loci with $K=10^{10}$

Stepwise locus ordering results in a list of the locus orders with the largest maximum likelihoods. To construct a framework map of loci, we began with those loci whose positions were consistent among all locus orders with maximum likelihoods no more than 1000 times smaller than that of the best locus order. We first noted whether these loci could be ordered at 1000:1. To those loci that could, we added any other loci whose best position gave a locus order maximum likelihood at least 1000 times greater than their next best position. Given the choice of several loci to include in a framework map, loci present in other genetic and physical maps of this region were given preference.

DNA probes. DNA markers used in this study are listed in Table 1. Plasmid DNA was prepared by standard techniques; prior to use as 
probes, DNA fragments were isolated from agarose gels by electrolution (Maniatis et al., 1982).

Standard and pulsed-field Southern hybridizations. For standard hybridizations, DNA was extracted from hybrid cells, digested to completion using EcoRI or HindIII, electrophoresed in 1\% agarose in $4 \times$ TAE, transferred to Hybond nylon membranes (Amersham), hybridized, and washed as described (Gorski et al., 1991). Radioactive probes were prepared by random oligonucleotide priming (Feinberg and Vogelstein, 1983). To score RHs for the presence or absence of DNA markers and control for the quantity of DNA present in each lane, 2 to 3 probes were pooled; each pool included a probe which detected distinct, evolutionarily conserved sequences in hamster DNA. Hybridizations were performed for $16-24 \mathrm{~h}$ at $65^{\circ} \mathrm{C}$ and washed to a final stringency of $0.1 \times \mathrm{SSC}, 0.1 \% \mathrm{SDS}$ at $65^{\circ} \mathrm{C}$ for at least $15 \mathrm{~min}$.

The methods for the preparation of agarose blocks containing highmolecular-weight DNA, the preparation of yeast and bacteriophage lambda multimers used as size markers, restriction digests, transfers to Hybond membranes, and hybridizations were described (Gorski et al., 1991). PFGE was performed at $14^{\circ} \mathrm{C}$ in $0.5 \times \mathrm{TBE}$ buffer (Maniatis et al., 1982) using a contour-clamped homogeneous electrical field (CHEF) gel system (Chu et al., 1986) (CHEF DRII, Bio-Rad). To determine whether two probes hybridized to the same restriction fragment, we employed different sets of electrophoresis conditions to shift the fragments into the regions of high resolution on the gel and sequentially hybridized probes to the same filter. Probes were removed from a filter prior to rehybridization; probe removal was verified by autoradiography.

Yeast artificial chromosomes. Using oligonucleotide primer pairs derived from loci mapped to Xp11.21, the Washington University yeast artificial chromosome (YAC) library was screened to identify yeast colonies containing cloned fragments; pooled YAC clones were screened using a PCR-based assay (Green and Olsen, 1990). To identify individual YAC clones, but circumvent the need for colony hybridizations, pooled yeast lysates of arrayed rows and columns were analyzed by PCR (Kwiatkowski et al., 1990). High-molecular-weight yeast DNA was prepared as previously described (Chandrasekharappa et al., 1992). Three YAC clones that contained loci mapped to a region between IP1 X-chromosomal translocation breakpoints within $\mathrm{Xp} 11.21$ were isolated; YAC clones B51B4 and B174A9 contained DXS14, and YAC clone B91F3 contained DXS370.

\section{RESULTS}

\section{Radiation Hybrids}

Radiation hybrid mapping was used in conjunction with physical mapping to determine the order and relative distances between 14 loci in the proximal region of human Xp. Sixty-seven RHs were isolated. Using a cloned human LINES repetitive DNA sequence as probe (Manuelidis and Biro, 1982), we found that $50 \mathrm{RHs}$ (75\%) contained a human-specific 1.9 -kb HindIII fragment (data not shown); this suggested that at least $75 \%$ of the RHs contained human X-chromosomal fragments (Cox et al., 1989). Since most of the RHs appeared to contain human DNA, all were analyzed; although RH 24 was not found to contain LINES sequences, it was found to contain human $\mathrm{X}$-chromosomal loci. The locus retention pattern for each of the RHs analyzed is summarized in Table 2. Limited quantities of DNA resulted in the incomplete scoring of $2 \mathrm{RHs}, \mathrm{RH} 49$ and $\mathrm{RH} 53$; all other RHs were completely scored. The retention frequency of the loci varied dramatically: $47 \mathrm{RHs}(70 \%)$ nonselectively retained centromeric DNA (DXZ1) but only 14 RHs (22\%) retained the OTC locus; other loci exhibited retention frequencies between these 2 values (Table 4). For each hybrid, the minimum number of obligate breaks required to explain the data ranged from zero to five (Table 2); an obligate break corresponds to a discordance in the presence $(+)$ or absence $(-)$ of adjacent typed loci (Boehnke et al., 1991).

\section{Multipoint Analysis of Radiation Hybrid Data}

We began our multipoint analysis by carrying out stepwise locus ordering for both the equal and the centromeric retention models. Both models gave the same best locus order. The centromeric model fit the data significantly better than the equal retention probability model $\left(\chi^{2}(1)=32.88, P<0.0001\right)$, consistent with our observation of decreasing retention as a function of distance from the centromere. Table 3 presents the 13 locus orders with maximum likelihoods no more than 1000 times less than that of the best locus order under the centromeric retention probability model; locus orders are presented only in their most likely orientation relative to the centromere. The most likely locus order and the distance estimates between loci under the centromeric retention probability model are summarized in Table 4 . To determine whether the data collected for the centromeric locus DXZ1 influenced the order of the other loci, multipoint analysis was performed without locus DXZ1 data; the absence of locus DXZ1 did not alter the resulting locus orders. Influential hybrids (Boehnke et al., 1991) and those requiring four or more obligate breaks were rescored. Two scores were changed; however, neither change significantly altered the resulting locus orders or distance estimates.

An interesting complication arose as we constructed a framework map from the list of orders in Table 3. From these orders, it appeared that the loci DXZ1, DXS14, MTHFDL1, DXS705, DXS741, ALAS2, DXS146, and OTC should be the basis for a 1000:1 framework map. However, when only these loci were analyzed, the relative positions of ALAS2, DXS705, and DXS741 could not be determined at that level of evidence. Because of the suggestion that DXS705 and DXS741 flank the IP1 locus (see Somatic Cell Hybrid Results), we have left all three orders in the near-framework maps of these eight loci (Table 5). In these maps, all loci but ALAS2, DXS705, and DXS741 are positioned with relative maximum likelihoods of at least 1000:1. The first nearframework map has a maximum likelihood 40 and 58 times greater than those of the second and the third, respectively, suggesting that it is the correct order, although not at 1000:1 relative likelihood.

\section{Somatic Cell Hybrid Mapping Panel Results}

To assign the 14 loci to distinct physical intervals within Xp, each probe was hybridized to blots containing IP1 somatic cell hybrid mapping panel DNA. As indicated in Table 1, by using this mapping panel, most of these loci have been previously mapped to specific intervals within Xp (Gorski et al., 1991, 1992). The results of 
TABLE 2

Locus Retention for the Best Locus Order

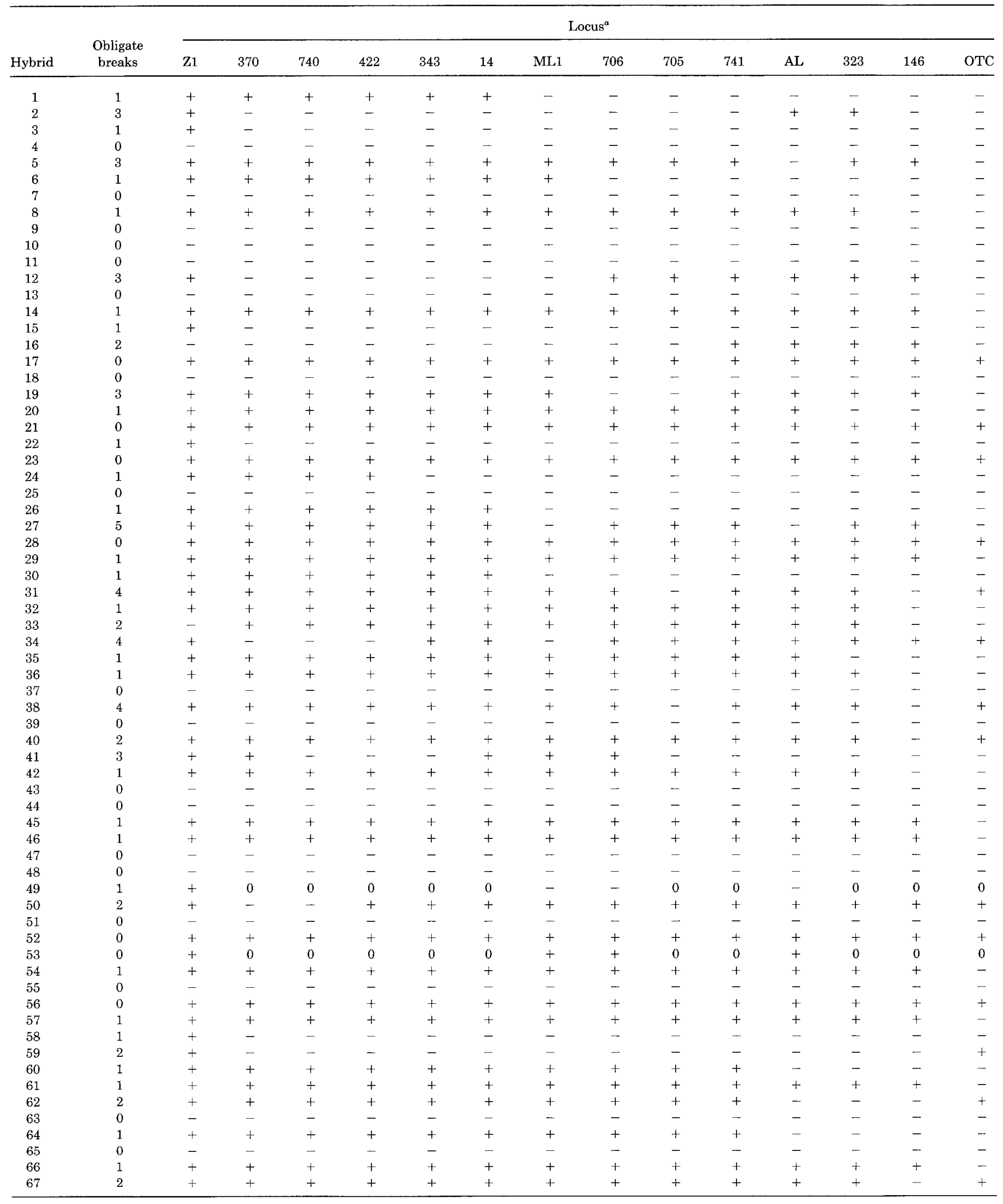

Note. The symbols,+- , and 0 indicate locus typed and present, locus typed and absent, and locus not typed, respectively. ${ }^{a}$ All numbered loci have DXS as prefix; Z1, DXZ1; ML1, MTHFDL1; AL, ALAS2. 
TABLE 3

Most Likely Locus Orders for the Centromere Retention Model

\begin{tabular}{|c|c|c|c|c|c|c|c|c|c|c|c|c|c|c|c|c|}
\hline Rank & $\begin{array}{l}\text { Likelihood } \\
\text { ratio }\end{array}$ & $\begin{array}{c}\text { Obligate } \\
\text { breaks }\end{array}$ & & & & & & & Locus & $\operatorname{rder}^{a}$ & & & & & & \\
\hline 1 & 1 & 72 & $\mathrm{Z} 1$ & 370 & 740 & 422 & 343 & 14 & ML1 & 706 & 705 & 741 & $\mathrm{AL}$ & 323 & 146 & OTC \\
\hline 2 & 2 & 72 & $\mathrm{Z} 1$ & 14 & 343 & 422 & 740 & 370 & ML1 & 706 & 705 & 741 & $\mathrm{AL}$ & 323 & 146 & OTC \\
\hline 3 & 60 & 74 & $\mathrm{Z1}$ & 370 & 740 & 422 & 343 & 14 & ML1 & 705 & 706 & 741 & $\mathrm{AL}$ & 323 & 146 & OTC \\
\hline 4 & 101 & 74 & $\mathrm{Z} 1$ & 14 & 343 & 422 & 740 & 370 & ML1 & 705 & 706 & 741 & $A L$ & 323 & 146 & OTC \\
\hline 5 & 354 & 74 & $\mathrm{Z} 1$ & 740 & 370 & 422 & 343 & 14 & ML1 & 706 & 705 & 741 & $\mathrm{AL}$ & 323 & 146 & OTC \\
\hline 6 & 402 & 74 & $\mathrm{Z} 1$ & 343 & 14 & 422 & 740 & 370 & ML1 & 706 & 705 & 741 & $\mathrm{AL}$ & 323 & 146 & OTC \\
\hline 7 & 402 & 74 & $\mathrm{Z} 1$ & 343 & 14 & 370 & 740 & 422 & ML1 & 706 & 705 & 741 & $\mathrm{AL}$ & 323 & 146 & OTC \\
\hline 8 & 402 & 74 & $\mathrm{Z} 1$ & 422 & 740 & 370 & 14 & 343 & ML1 & 706 & 705 & 741 & $\mathrm{AL}$ & 323 & 146 & OTC \\
\hline 9 & 402 & 74 & $\mathrm{Z} 1$ & 370 & 740 & 422 & 14 & 343 & ML1 & 706 & 705 & 741 & $\mathrm{AL}$ & 323 & 146 & OTC \\
\hline 10 & 536 & 76 & $\mathrm{Z1}$ & 370 & 740 & 422 & 343 & 14 & ML1 & 706 & 705 & 741 & 323 & $\mathrm{AL}$ & 146 & OTC \\
\hline 11 & 679 & 74 & $\mathrm{Z1}$ & 422 & 740 & 370 & 343 & 14 & ML1 & 706 & 705 & 741 & $\mathrm{AL}$ & 323 & 146 & OTC \\
\hline 12 & 901 & 76 & $\mathrm{Z1}$ & 14 & 343 & 422 & 740 & 370 & ML1 & 706 & 705 & 741 & 323 & $\mathrm{AL}$ & 146 & OTC \\
\hline 13 & 931 & 74 & $\mathrm{Z1}$ & 14 & 343 & 422 & 370 & 740 & ML1 & 706 & 705 & 741 & AL & 323 & 146 & OTC \\
\hline
\end{tabular}

Note. Likelihood ratio compares the maximum likelihood for the given locus order to that for the overall maximum likelihood order.

${ }^{a}$ Single underlines indicate inversions of two or more loci relative to the best locus order; double underlines indicate more complex rearrangements.

these analyses are in total agreement with all of the most likely locus orders (Table 3 ) and with the first of the near-framework locus orders (Table 5). Loci DXS14, DXS370, DXS422, DXS343, DXS740, DXS705, DXS 706 , and MTHFDL1 mapped to a region between IP1 $\mathrm{X}$-chromosomal translocation breakpoints; these markers were distal to the IP1 X;17 breakpoint (cell line C171D3) and proximal to the IP1 $X ; 13$ and $X ; 9$ breakpoints (cell lines B13-3 and C9-5, respectively). Loci DXS323, DXS741, and ALAS2 were distal to the IP1 X;13 and $\mathrm{X} ; 9$ breakpoints and proximal to the translocation breakpoint contained in cell line GM10501; locus DXS146 was distal to the GM10501 breakpoint. These results are summarized schematically in a companion paper (Gorski et al., 1992). In combination, the somatic cell hybrid and radiation hybrid mapping results indicate that the $\mathrm{X} ; 13$ and X;9 IP1 X-chromosomal translocation breakpoints are flanked by loci DXS705 and DXS741 and that these loci are separated by approximately $12 \mathrm{cR}$ (Table 4).

\section{Molecular Mapping}

As shown in Table 3, our analysis of the radiation hybrid data was unable to provide a definitive order for the five loci, DXS14, DXS370, DXS422, DXS740, and DXS343. We had previously used PFGE analyses to identify overlapping restriction fragments detected by probes cpX210, MTHFDL1, and 3S. These results showed that probes $\mathrm{cpX} 210$ and MTHFDL1 were within $660 \mathrm{~kb}$ and that probes MTHFDL1 and 3S were within $1200 \mathrm{~kb}$ and suggested the order: DXS422-MTHFDL1DXS705 (Gorski et al., 1992). Combined with our parameter estimates (Table 4), the PFGE data provided an approximate size for a $\mathrm{cR}_{8000}(1 \mathrm{cR}=26 \mathrm{~kb})$. Since the five unordered loci were estimated to be within $15 \mathrm{cR}$, our data suggested that all five were within approximately $390 \mathrm{~kb}$; we performed physical mapping experiments to determine the accuracy of these estimated distances.

Using primers derived from loci DXS14 and DXS370, three YAC clones were isolated: a $230-$ and a $350-\mathrm{kb}$ YAC clone, B51B4 and B174A9, respectively, were isolated using DXS14-derived primers; a 260-kb YAC clone, B91F3, was isolated using DXS370-derived primers. Four of the five loci were found to be contained within these three YACs. Probe 49A hybridized to both B51B4 and B174A9; probe RX-66 hybridized only to B174A9. As expected, probes p58-1 and $30 \mathrm{C}_{\mathrm{E}} 2.8$ hybridized to their respective YACs; neither probe hybridized to additional YACs (Fig. 1). Probe cpX210 did not hybridize to any of the YACs (data not shown). These results showed that DXS14, DXS740, and DXS343 are within $350 \mathrm{~kb}$.

PFGE analyses were performed to provide an estimated distance between the remaining loci. We have previously shown that DXS14 and DXS422 are within 125 kb (Gorski et al., 1991). As shown in Fig. 2, probes p58-1 and $30 \mathrm{C}_{\mathrm{E}} 2.8$ detect superimposable $S a c \mathrm{II}, S f i \mathrm{I}$, and $E a g \mathrm{I}$ fragments; these results map DXS14 and DXS370 to within $350 \mathrm{~kb}$. Although these results do not provide a definitive locus order, they support the localization and the distance estimates provided by the radiation hybrid mapping analyses.

\section{DISCUSSION}

The order of and relative distances between 14 DNA loci in the proximal short arm of the human $\mathrm{X}$ chromo- 
TABLE 4

Parameter Estimates for the Most Likely Locus Order

\begin{tabular}{|c|c|c|c|c|}
\hline Locus & $\begin{array}{l}\text { Breakage probability } \\
\text { estimate }(\theta)\end{array}$ & $\begin{array}{l}\text { Distance estimate } \\
\text { (cR) }\end{array}$ & $\begin{array}{l}\text { Observed retention } \\
\text { frequency }(\%)\end{array}$ & $\begin{array}{c}\text { Estimated retention } \\
\text { probability (\%) }\end{array}$ \\
\hline \multirow[t]{2}{*}{$\mathrm{Z} 1$} & & & 70.1 & 70.1 \\
\hline & 0.263 & 30.5 & & \\
\hline \multirow[t]{2}{*}{370} & & & 56.9 & 58.2 \\
\hline & 0.029 & 3.0 & & \\
\hline \multirow{2}{*}{740} & & & 55.4 & 57.2 \\
\hline & 0.029 & 3.0 & & \\
\hline \multirow[t]{2}{*}{422} & & & 56.9 & 56.2 \\
\hline & 0.058 & 6.0 & & \\
\hline \multirow[t]{2}{*}{343} & & & 56.9 & 54.4 \\
\hline & 0.029 & 2.9 & & \\
\hline \multirow[t]{2}{*}{14} & & & 58.5 & 53.6 \\
\hline & 0.146 & 15.8 & & \\
\hline \multirow[t]{2}{*}{ ML1 } & & & 50.7 & 49.3 \\
\hline & 0.144 & 15.6 & & \\
\hline \multirow[t]{2}{*}{706} & & & 52.2 & 45.8 \\
\hline & 0.089 & 9.3 & & \\
\hline \multirow[t]{2}{*}{705} & & & 47.7 & 43.9 \\
\hline & 0.117 & 12.4 & & \\
\hline \multirow[t]{2}{*}{741} & & & 53.8 & 41.6 \\
\hline & 0.174 & 19.1 & & \\
\hline \multirow[t]{2}{*}{$\mathrm{AL}$} & & & 47.8 & 38.7 \\
\hline & 0.124 & 13.2 & & \\
\hline \multirow[t]{2}{*}{323} & & & 47.7 & 36.9 \\
\hline & 0.331 & 40.2 & & \\
\hline \multirow[t]{2}{*}{146} & & & 32.3 & 32.8 \\
\hline & 0.750 & 138.4 & & \\
\hline OTC & & & 21.5 & 26.7 \\
\hline
\end{tabular}

Note. Distance estimates were calculated using the distance transformation $D=-\log (1-\theta)$. The estimated retention probabilities were calculated using the centromeric retention probability model (Boehnke et al., 1991).

some were determined by a combination of radiation hy- cosegregation of DNA markers in radiation hybrid brid and physical mapping techniques. A multipoint clones and construct a 14-locus comprehensive map and maximum likelihood method was used to analyze the three 8-locus near-framework maps that extend from

TABLE 5

\section{Near-Framework Maps of Proximal Xp}

Rank Likelihood ratio

Note. Distance estimates $(D)$ are provided as centirays (cR). The total map length for the best order is $301.7 \mathrm{cR}$; the total map lengths for the second and third orders are 309.8 and $309.5 \mathrm{cR}$, respectively. 


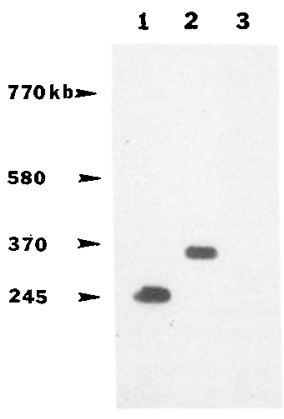

49A
12

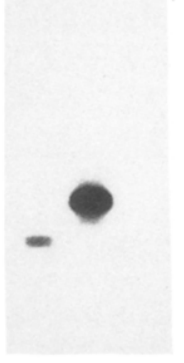

p58-1

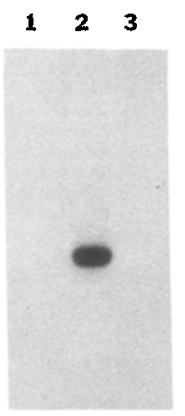

RX-66
123

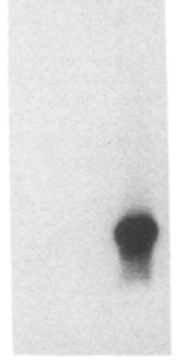

$30 C_{E} 2.8$
FIG. 1. Southern blot analysis of duplicate filters containing similarly loaded high-molecular-weight yeast DNA; YAC clone DNA containing either locus DXS14 (clones B51B4, lane 1; and B174A9, lane 2) or locus DXS370 (clone B91F3, lane 3), hybridized with probes 49A, p58-1, RX-66, and $30 \mathrm{C}_{\mathrm{E}} 2.8$. DNA was fractionated by $\mathrm{CHEF}$ electrophoresis in $1.0 \%$ agarose $(0.5 \times \mathrm{TBE})$ using $200 \mathrm{~V}$ at $14^{\circ} \mathrm{C}$ with switch times of $60 \mathrm{~s}$ for $15 \mathrm{~h}$ and a $90 \mathrm{~s}$ for $9 \mathrm{~h}$. Probes p58-1 and 49A hybridized to both the $230-\mathrm{kb}$ YAC clone B51B4 and the $350-\mathrm{kb}$ YAC clone B174A9; probe RX-66 hybridized only to clone B174A9; and probe $30 \mathrm{C}_{\mathrm{E}} 2.8$ hybridized to only the $260 \mathrm{~kb}$ YAC clone B91F3.

Xp11.4 to Xcen. Radiation hybrid mapping results were verified by using a somatic cell hybrid mapping panel of naturally occurring $\mathrm{X}$; autosome translocations and PFGE analysis; no discrepancies of locus order or distance were identified. All of the constructed radiation hybrids were used to construct the map without prior screening to identify those hybrids containing human repetitive sequence elements. Our data and previous analyses have shown that screening may fail to identify all hybrids containing human fragments (Warrington et al., 1991). No bias was introduced to preselect for hybrids containing larger fragments or larger quantities of human repetitive sequences.

Our results illustrate that, for a human $\mathrm{X}$ chromosome irradiated with 8000 rads, 67 hybrids were suffi- cient to construct a useful map; the most detailed portion of the map spans an estimated physical distance of approximately $2400 \mathrm{~kb}$ and includes 12 loci within or flanking region Xp11.21. Our physical calibration of radiation map distances $(1 \mathrm{cR}=26 \mathrm{~kb})$ is comparable to previous estimates with similar doses of radiation (Cox et al., 1990; Warrington et al., 1991); given the uncertainties of the radiation dose actually applied and the actual PFGE distances measured, these differences may not be real.

Although this map was sufficient to identify closely spaced loci (those within $350 \mathrm{~kb}$ ), we were limited in our ability to order such loci. However, these loci were within the resolution of molecular mapping techniques such as YAC mapping and PFGE. These results illustrate the point that, although there will be a limit to the resolution of a radiation hybrid map, this method will serve to focus attention on tightly clustered loci. The resolution limits of radiation hybrid mapping will be influenced by numerous factors including: the number of hybrids analyzed, the error rate of analysis, the distance between markers, and locus retention frequency; it is likely that the high retention frequencies of the loci scored favorably influenced the resolution of the constructed radiation hybrid map.

In the course of constructing the radiation hybrid map, we encountered several unexpected results. First, a remarkable locus retention frequency gradient was observed; loci at or near the centromere were retained with a much higher frequency than those mapped distally. Similar, but smaller gradients have been observed in other radiation hybrid panels (Benham et al., 1989; Cox et al., 1990; Richard et al., 1991; Ceccherini et al., 1992). These observations suggest that during the construction and/or passage of radiation hybrids, centromeric DNA may be preferentially retained. It is possible that such
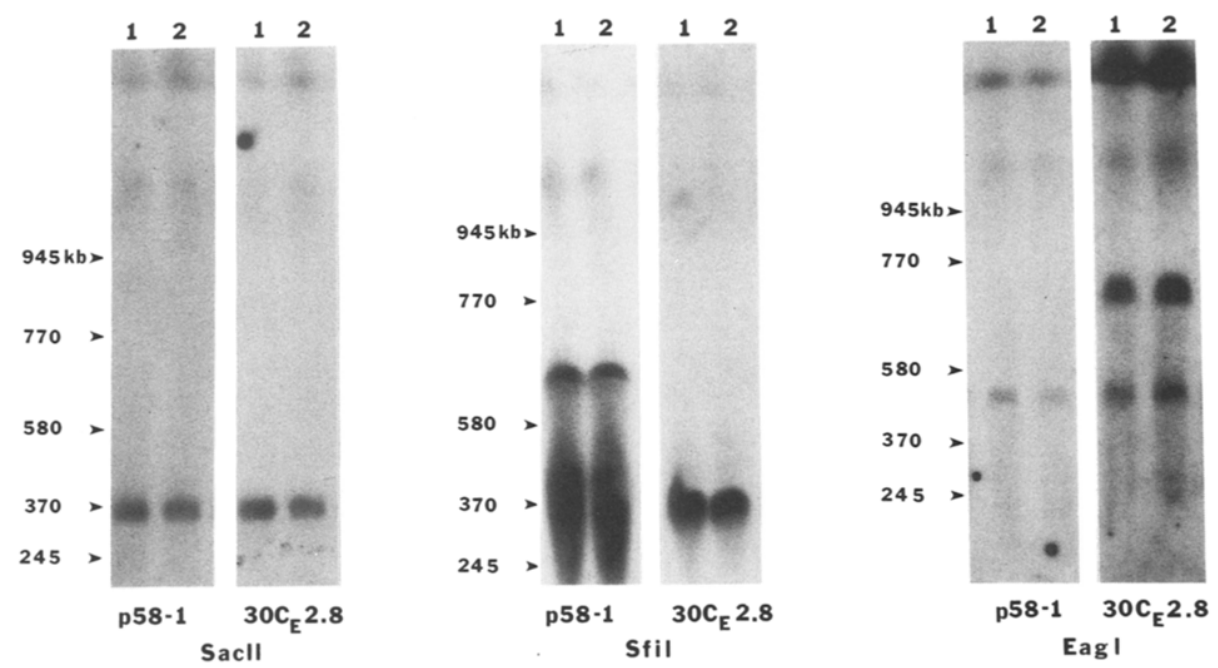

FIG. 2. Southern blot analysis of X-only somatic cell hybrid DNA partially digested with either 15 U (lane 1) or 5 U (lane 2) of SacII, Sfi I, or EagI; the same filters were sequentially hybridized with probes p58-1 and $30 \mathrm{C}_{\mathrm{E}} 2.8$. DNA was fractionated by CHEF electrophoresis in $1.0 \%$ agarose $(0.5 \times \mathrm{TBE})$ using $200 \mathrm{~V}$ at $14^{\circ} \mathrm{C}$. Switch times were $60 \mathrm{~s}$ for $15 \mathrm{~h}$ and $120 \mathrm{~s}$ for $12 \mathrm{~h}$ for $S a c I I, 60 \mathrm{~s}$ for $20 \mathrm{~h}$ and $120 \mathrm{~s}$ for $12 \mathrm{~h}$ for $S f \mathrm{I}$, and $60 \mathrm{~s}$ for $18 \mathrm{~h}$ and $120 \mathrm{~s}$ for $12 \mathrm{~h}$ for EagI. Probes p58-1 and $30 \mathrm{C}_{\mathrm{E}} 2.8$ detected superimposable 350-kb SacII, 350-kb SfI, and 500-kb EagI fragments. Probe p58-1 detected additional 400- and 660-kb SfiI fragments, and probe $30 \mathrm{C}_{\mathrm{E}} 2.8$ detected an additional 710 -kb EagI fragment; using a variety of parameters, previous hybridizations with probe p58-1 identified a 580-kb Sfil fragment (Gorski et al., 1991). 
preferential retention may be secondary to fundamental differences between centromeric and non-centromeric DNA; potential differences in susceptibilities to degradation after fragmentation, integration frequencies, or mitotic stabilities might influence retention frequency. Since centromeric DNA was retained more frequently, it was possible that our analyses would be confused by radiation hybrids containing frequent, independently segregating centromeric fragments. We did not, however, encounter any ambiguities in the localization of the centromeric DNA, nor did the removal of the centromeric locus data alter our mapping results. It is possible that this potential problem was circumvented by not using randomly selected X-chromosomal loci to score the hybrids; all loci used to score the hybrids had been previously mapped to an interval in close proximity to the centromere. This suggests that radiation hybrid mapping will be facilitated by using markers previously assigned to chromosomal intervals.

Second, during the construction of the near-framework maps, it was unanticipated that the removal of several loci from the multipoint maximum likelihood analysis would affect the likelihood of the orders of other nonadjacent loci; this suggests that each locus analyzed might potentially alter previous estimates of position and distance. It remains to be determined with what frequency and at what resolution such additional breaks and subsequent amended maps might occur. Obviously, undetected data errors may play a role in this observation.

The radiation hybrid mapping panel reported here will provide a rapid mapping tool for any identified DNA marker localized to proximal Xp. Once a DNA marker is available, it will be possible to place a DNA sequence relative to mapped markers in several days. The radiation hybrid mapping panel also provides a way of identifying groups of loci that are close enough to warrant subsequent analyses with techniques yielding high-resolution physical maps such as PFGE, YAC clone analysis (Schlessinger et al., 1991), and multicolor interphase fluorescent in situ hybridization (Trask et al., 1991).

Most importantly, this map provides a means for identifying markers in close proximity to disease loci and disease-specific X-chromosomal rearrangements. This map has localized a candidate gene for sideroblastic anemia (ALAS2) (Cotter et al., 1992) and a pseudogene (MTHFDL1) (Italiano et $a l$., 1991) and has identified DNA markers that flank the IP1 X-chromosomal translocation breakpoints. These results have provided a new focus for our IP1 cloning efforts; without radiation hybrid mapping, there would be no compelling reason to focus on a particular pair of loci for subsequent high-resolution mapping studies. We are currently screening YAC libraries in an attempt to establish a cloned YAC contig spanning the IP1 translocation breakpoints. Several additional disease-related loci have been mapped to proximal Xp including: progressive cone dystrophy, Wiskott Aldridge disease, Norries disease, retinitis pigmentosa types 2 and 3 , congenital stationary night blindness, and an X-chromosomal translocation break- point associated with synovial sarcoma (Davies et al., 1990); this map and the radiation hybrid panel may be of utility in mapping these loci.

\section{ACKNOWLEDGMENTS}

We thank D. Cox and F. Collins for stimulating conversations and helpful comments; D. Barker, D. Bishop, G. Bruns, K. Davies, T. Kruse, R. Rozen, S. Weissman, and H. Willard, for cloned human DNA probes; $\mathrm{H}$. Willard for somatic cell hybrids A48-1Fa and A631A; and D. Voss for assisting in preparing the manuscript. Cell lines GM06853, GM10501, and GM06318 were obtained from the NIGMS Human Genetic Mutant Cell Repository, Coriell Institute of Medical Research (Camden, NJ). This work was supported, in part, by March of Dimes-Birth Defects Foundation Basic Science Grant 1-91-176 and National Institutes of Health (NIH) Grants HD-23768 and NS30771 to J.L.G., NIH Training Grant NIH-5-T32-GM07544-11 to E.N.B., and The University of Michigan Human Genome Center P30HG00209.

\section{REFERENCES}

Arenstorf, H. P., Kandpal, R. P., Baskaran, N., Parimoo, S., Tanaka, Y., Kitajima, S., Yasukochi, Y., and Weissman, S. M. (1991). Construction and characterization of a NotI-BsuE linking library from the human X chromosome. Genomics 11: 115-123.

Benham, F., Hart, K., Crolla, J., Bobrow, M., Francavilla, M., and Goodfellow, P. N. (1989). A method for generating hybrids containing nonselected fragments of human chromosomes. Genomics 4: 509-517.

Boehnke, M., Lange, K., and Cox, D. R. (1991). Statistical methods for multipoint radiation hybrid mapping. Am. J. Hum. Genet. 49: 1174-1188.

Burright, E. N., and Gorski, J. L. (1992). Isolation of Alu-PCR products from a $660-\mathrm{kb} S f \mathrm{I}$ restriction fragment mapped to a region between incontinentia pigmenti 1 (IP1) X-chromosomal translocation breakpoints. Submitted for publication.

Cannizzaro, L. A., and Hecht, F. (1987). Gene for incontinentia pigmenti maps to band Xp11.21 with an $(\mathrm{X} ; 10)(\mathrm{p} 11 ; \mathrm{q} 22)$ translocation. Clin. Genet. 32: 66-69.

Ceccherini, I., Romeo, G., Lawrence, S., Breuning, M. H., Harris, P. C., Himmelbauer, H., Frischauf, A. M., Sutherland, G. R., Germino, G. G., Reeders, S. T., and Morton, N. E. (1992). Construction of a map of chromosome 16 by using radiation hybrids. Proc. Natl. Acad. Sci. USA 89: 104-108.

Chandrasekharappa, S. C., Marchuk, D. A., and Collins, F. S. (1992). Analysis of yeast artificial chromosome clones. In "Methods in Mo lecular Biology: Pulsed Gel Electrophoresis Techniques" (L. Ulanovsky and M. Burmeister, Eds.), Humana Press, Clifton, NJ, in press.

Chu, G., Vollrath, D., and Davis, R. W. (1986). Separation of large DNA molecules by contour-clamped homogeneous electric fields. Science 234: 1582-1585.

Cotter, P. D., Willard, H. F., Gorski, J. L., and Bishop, D. F. (1992). Assignment of human erythroid delta-aminolevulinate synthase (ALAS2) to a distal subregion of band $\mathrm{Xp11.21} \mathrm{by} \mathrm{PCR} \mathrm{analysis} \mathrm{of}$ somatic cell hybrids containing X; autosome translocations. Genomics 13: $211-213$.

Cox, D. R., Pritchard, C. A., Uglum, E., Casher, D., Kobori, J., and Myers, R. M. (1989). Segregation of the Huntington disease region of human chromosome 4 in a somatic cell hybrid. Genomics 4: 397407.

Cox, D. R., Burmeister, M., Price, E. R., Kim, S., and Myers, R. M. (1990). Radiation hybrid mapping: A somatic cell genetic method for constructing high-resolution maps of mammalian chromosomes. Science 250: 245-250.

Crolla, J. A., Gilgenkrantz, S., de Grouchy, J., Kajii, T., and Bobrow, 
M. (1989). Incontinentia pigmenti and X-autosome translocations: Non-isotopic in situ hybridization with an X-centromere-specific probe (pSV2X5) reveals a possible X-centromeric breakpoint in one of five published cases. Hum. Genet. 81: 269-272.

Davies, K. E., Mandel, J. L., Monaco, A. P., Nussbaum, R. L., and Willard, H. F. (1990). Report of the committee on the genetic constitution of the X chromosome. Cytogenet. Cell Genet. 55: 254-313.

Feinberg, A., and Vogelstein, B. (1983). A technique for radiolabeling DNA restriction endonuclease fragments to high specific activity. Anal. Biochem. 132: 6-13.

Gilgenkrantz, S., Tridon, P., Pinel-Briquel, N., Beurey, J., and Weber, M. (1985). Translocation $(X ; 9)(\mathrm{p} 11 ; \mathrm{q} 34)$ in a girl with incontinentia pigmenti (IP): Implications for the regional assignment of the IP locus to Xp11. Ann. Genet. 28: 90-92.

Gorski, J. L., Stein, C. K., and Glover, T. W. (1989). A somatic cell hybrid panel to facilitate identification of DNA sequences in the vicinity of the incontinentia pigmenti locus (IP1). Cytogenet. Cell Genet. 52: 90-92.

Gorski, J. L., Burright, E. N., Harnden, C. E., Stein, C. K., Glover, T. W., and Reyner, E. L. (1991). Localization of DNA sequences to a region within Xp11.21 between incontinentia pigmenti (IP1) Xchromosomal translocation breakpoints. Am. J. Hum. Genet. 48: 53-64.

Gorski, J. L., Burright, E. N., Reyner, E. L., Goodfellow, P. N., and Burgess, D. L. (1992). Isolation of DNA markers from a region between incontinentia pigmenti 1 (IP1) X-chromosomal translocation breakpoints by a comparative PCR analysis of a radiation hybrid subclone mapping panel. Genomics 14: $649-656$

Green, E. D., and Olson, M. V. (1990). Systematic screening of yeast artificial-chromosome libraries by use of the polymerase chain reaction. Proc. Natl. Acad. Sci. USA 87: 1213-1217.

Harnden, D. G., and Klinger, H. P., Eds. (1985). "An International System for Human Cytogenetic Nomenclature," ISCN, published in collaboration with Cytogenet. Cell Genet., Karger, Basel.

Hodgson, S. V., Neville, B., Jones, R. W. A., Fear, C., and Bobrow, M. (1985). Two cases of $\mathrm{X}$; autosome translocation in females with incontinentia pigmenti. Hum. Genet. 71: 231-234.

Italiano, C., John, S. W. M., Hum, D. W., Mackenzie, R. E., and Rozen, R. (1991). A pseudogene on the $X$ chromosome for the human trifunctional enzyme MTHFD (methylenetetrahydrofolate dehy. drogenase-methenyltetrahydrofolate cyclohydrolase-formyltetrahydrofolate synthetase). Genomics 10: 1073-1074.
Kajii, T., Tsukahara, M., Fukushima, Y., Hata, A., Matsuo, K., and Kuroki, Y. (1985). Translocation (X;13)(p11.21;q12.3) in a girl with incontinentia pigmenti and bilateral retinoblastoma. Ann. Genet. 28: 219-223.

Kwiatkowski, T. J., Jr., Zoghbi, H. Y., Ledbetter, S. A., Ellison, K. A., and Chinault, A. C. (1990). Rapid identification of yeast artificial chromosome clones by matrix pooling and crude lysate PCR. $\mathrm{Nu}$ cleic Acids Res. 18: 7191-7192.

Lafreniere, R. G., Brown, C. J., Powers, V. E., Carrel, L., Davies, K. E., Barker, D. F., and Willard, H. F. (1991). Physical mapping of 60 DNA markers in the p21.1-q21.3 region of the human X chromosome. Genomics 11: 352-363.

Mahtani, M. M., and Willard, H. F. (1988). A primary genetic map of the pericentric region of the human $\mathrm{X}$ chromosome. Genomics 2: 294-301.

Maniatis, T., Fritsch, E. F., and Sambrook, J. (1982). "Molecular Cloning: A Laboratory Manual," Cold Spring Harbor Laboratory, Cold Spring Harbor, NY.

Manuelidis, L., and Biro, P. A. (1982). Genomic representation of the HindIII $1.9 \mathrm{~kb}$ repeated DNA. Nucleic Acids Res. 10: 3221-3239.

Richard, C. W., III, Withers, D. A., Meeker, T. C., Maurer, S., Evans, G. A., Myers, R. M., and Cox, D. R. (1991). A radiation hybrid map of the proximal long arm of human chromosome 11 containing the multiple endocrine neoplasia type 1 (MEN-I) and $b c l$-I disease loci. Am. J. Hum. Genet. 49: 1189-1196.

Schlessinger, D., Little, R. D., Freije, D., Abidi, F., Zucchi, I., Porta, G., Pilia, G., Nagaraja, R., Johnson, S. K., Yoon, J-Y., Srivastava, A., Kere, J., Palmieri, G., Ciccodicola, A., Montanaro, V., Romano, G., Casamassimi, A., and D'Urso, M. (1991). Yeast artificial chromosome-based genome mapping: Some lessons from Xq24-q28. Genomics 11: 783-793.

Trask, B. J., Massa, H., Kenwrick, S., and Gitschier, J. (1991). Mapping of human chromosome Xq28 by two-color fluorescence in situ hybridization of DNA sequences to interphase cell nuclei. Am. J. Hum. Genet. 48: 1-15.

Warrington, J. A., Hall, L. V., Hinton, L. M., Miller, J. N., Wasmuth, J. J., and Lovett, M. (1991). Radiation hybrid map of 13 loci on the long arm of chromosome 5. Genomics 11: 701-708.

Westerveld, A., Visser, R. P. L. S., Kahn, P. M., and Bootsma, D. (1971). Loss of human genetic markers in man-Chinese hamster ovary cells. Nature (New Biol.) 234: 20-22. 